\title{
Éditorial: Énoncé de politique sur les appareils et accessoires fonctionnels pour personnes âgées
}

Énoncé du problème: Le Canada n'a pas de politique cohérente en matière de soutien à caractère technologique pour les personnes qui ont un handicap et/ou sont limitées sur le plan physique, ni en matière d'aide financière pour faciliter l'acquisition de tels appareils. Selon les études, le financement est un obstacle majeur à l'utilisation des appareils et accessoires fonctionnels (LaPlante, Hendershot, \& Moss, 1997; Kozarevic \& Israel, 1987). À cause de ce manque de fonds, il n'existe pas d'exigences de rapport systématique sur les appareils et accessoires fonctionnels entre programmes de services directs, ni de classification uniforme des dépenses engagées en appareils et accessoires fonctionnels, et la distinction qui est faite entre cette technologie particulière et la technologie courante est floue (Mendelsohn, 1997).

Portée: Par appareils et accessoires fonctionnels, on entend "tout article, appareil ou produit, acheté en magasin, modifié ou adapté, que l'on utilise pour augmenter, maintenir ou améliorer les capacités fonctionnelles des personnes ayant un handicap" (U.S. Technology Related Assistance for Individuals with Disabilities Act of 1988, Tech Act, 29 USC Sec.2201). Les catégories d'appareils et accessoires fonctionnels utilisées par les personnes âgées comprennent les aides à la mobilité, aux soins personnels, aux aptitudes domestiques et aux activités de loisirs (Axell \& Yasuda, 1993). Il n'existe pas assez de preuves venant appuyer ou réfuter l'argument selon lequel les appareils et accessoires fonctionnels permettent aux personnes âgées d'être plus autonomes. On sait pourtant que ce type d'appareils est couramment utilisé par les aînés ${ }^{1}$ puisque près de 35 pour cent des Canadiens et Canadiennes âgées de 75 ans et plus en utilisent au moins un, que 90 pour cent d'entre eux se servent d'aides à la vision et que, après 50 ans, le recours aux aides à la mobilité et à l'audition augmente respectivement de près de 50 et 60 pour cent (Forbes, Hayward, \& Agwani, 1993).

Analyse des résultats d'études: Le fait que, souvent, les personnes âgées ayant besoin d'appareils et accessoires fonctionnels ne les reçoivent pas, ou ne les utilisent pas, laisse supposer qu'on n'a pas bien expliqué que cet appareillage pourrait les rendre plus autonomes. La non-utilisation et/ou sous-utilisation des appareils et accessoires fonctionnels par ceux qui en ont besoin sont associées à des facteurs tels que la sous-scolarisation 
(Forbes et al., 1993), le fait d'habiter en région rurale (Forbes et al., 1993; Kozarevic \& Israel, 1987), l'isolement social (Forbes et al., 1993), le fait d'être une femme (Forbes et al., 1993; Kozarevic \& Israel, 1987), l'appartenance à des populations traditionnellement mal desservies par les systèmes de santé et de services sociaux (comme les autochtones et les résidents des régions rurales) (Galvin, 1997), et un âge avancé (Kozarevic \& Israel, 1987). Il est toutefois important de noter que, selon les études réalisées, 79 pour cent des personnes qui ont un appareil ou accessoire fonctionnel disent l'utiliser (Mann, Hurren, \& Tomita, 1993).

D'après l'une des plus grandes enquêtes américaines effectuées sur le sujet (National Council on Disability, 1993), 80 pour cent des personnes âgées déclarent être moins dépendantes des autres et près de 50 pour cent disent avoir pu éviter la maison de soins infirmiers parce qu'elles utilisaient un appareil ou accessoire fonctionnel. Selon la seule étude aléatoire et contrôlée relevée dans la documentation spécialisée, qui portait sur 22 aînés pensionnaires de maisons de soins infirmiers, ceux qui étaient équipés et savaient se servir d'une commande à distance manuelle pour allumer et éteindre la radio et la lumière écoutaient trois fois plus la radio que les autres personnes faisant partie de l'étude, ce qui laisse supposer qu'ils étaient plus autonomes (Mann, 1992).

L'utilisation qui est faite des appareils et accessoires fonctionnels ne permet pas nécessairement de prédire la perception qu'a chacun de sa propre autonomie. Cependant, si le nombre de personnes ayant ce type d'appareils et la fréquence avec laquelle elles s'en servent peuvent être considérées comme des mesures fiables, les appareils et accessoires fonctionnels, et notamment les aides à la mobilité, à l'audition et les dispositifs rendant le foyer accessible, semblent contribuer à l'autonomie fonctionnelle. Les personnes âgées seront plus enclines à utiliser des appareils et accessoires fonctionnels si cette utilisation est appuyée par des organismes de services (Mann et al., 1993).

Recommandations: L'Association canadienne de gérontologie appuie l'adoption par le gouvernement d'une définition des termes "appareils et accessoires fonctionnels" qui soit adaptée au contexte canadien et l'élaboration de politiques cohérentes visant l'octroi d'une aide financière aux personnes âgées pouvant bénéficier des avantages de cet appareillage.

L'Association canadienne de gérontologie encourage les bailleurs de fonds et les chercheurs à soutenir et entreprendre des recherches évaluatives approfondies pour étudier l'efficacité de la grande variété d'appareils et d'accessoires fonctionnels disponibles sur le marché.

L'Association canadienne de gérontologie est d'avis que d'importants progrès pourraient être réalisés si l'on adoptait le point de vue que les appareils et accessoires fonctionnels sont une façon de maximiser les capacités fonctionnelles et l'autonomie. 


\section{Note}

1 Le masculin embrasse le féminin.

\section{Références}

Axell, L.A., \& Yasuda, Y.L. (1993). Assistive devices and home modifications in geriatric rehabilitation. Geriatric Rehabilitation, 9, 803-821.

Forbes, W.F., Hayward, L.M., \& Agwani, N. (1993). Factors associated with self-reported use and non-use of assistive devices among impaired elderly residing in the community. Canadian Journal of Public Health, 84, 53-57.

Galvin, J.C. (1997). Consumerism and outreach to underrepresented populations. Technology and Disability, 6, 49-61.

Kozarevic, D.J., \& Israel, L. (1987). Disabilities and the level of affected activities of daily living. Revue épidémiologique et Santé publique, 35, 248-256.

LaPlante, M.P., Hendershot, G.E., \& Moss, A.J. (1997). The prevalence of need for assistive technology devices and home accessibility features. Technology and Disability, 6, 17-28.

Mann, W.C. (1992). Use of environmental control devices by elderly nursing home patients. Assistive Technology, 4, 60-65.

Mann, W.C., Hurren, D., \& Tomita, M. (1993). Comparison of assistive device use and needs of home-based older persons with different impairments. The American Journal of Occupational Therapy, 47, 980-987.

Mendelsohn, S.B. (1997). Assistive technology: public policy and financing. Technology and Disability, 6, 29-48.

National Council on Disability. (1993). Study on the financing of assistive technology devices and services for individuals with disabilities. Washington, DC.

Rédigé par Carol McWilliam, Ed.D., à la demande de conseil d'administration de l'Association canadienne de gérontologie, l'exposé ci-dessus constitute la position officielle du conseil adoptée le 26 octobre 2000. Cet énoncé est basé sur l'article "Care Delivery Approaches and Seniors' Independence" par C.L. McWilliam, W.L. Diehl-Jones, J. Jutai, et S. Tadrissi (La Revue canadienne du vieillissement (2000), 19 (suppl. 1), 101-124.). 